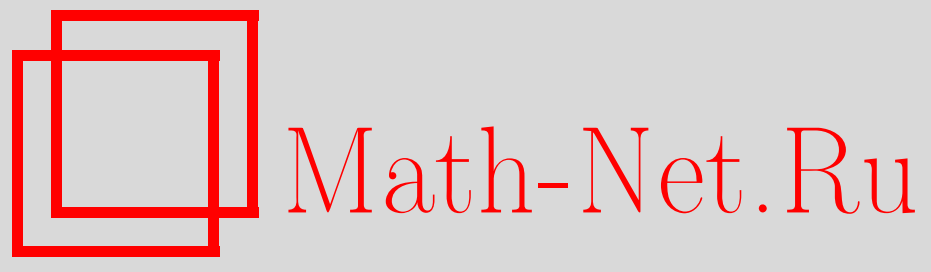

Ю. Е. Лозовик, А. Г. Семенов, К теории сверхтекучести в поляритонной системе, ТМФ, 2008, том 154, номер 2, 372-384

DOI: https://doi.org/10.4213/tmf6176

Использование Общероссийского математического портала Math-Net.Ru подразумевает, что вы прочитали и согласны с пользовательским соглашением http://www . mathnet.ru/rus/agreement

Параметры загрузки:

IP : 54.205 .225 .156

26 апреля 2023 г., 14:35:49

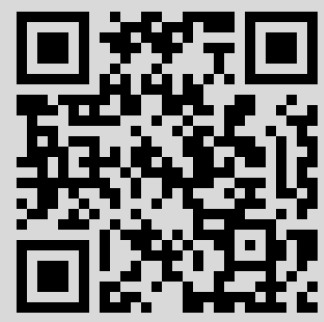




\title{
К ТЕОРИИ СВЕРХТЕКУЧЕСТИ В ПОЛЯРИТОННОЙ СИСТЕМЕ
}

\begin{abstract}
Рассматриваются сверхтекучие свойства двумерной системы поляритонов в оптической полости. С помощью вывода выражения для эффективного низкоэнергетического действия для термодинамических флуктуаций фазы получено выражение для аналога сверхтекучей плотности в системе в терминах корреляционной функции ток-ток, а также выражение для оператора тока. Описано приближение Боголюбова для поляритонной системы и вычислена сверхтекучая плотность. Обсуждается переход Березинского-Костерлица-Таулеса в исследуемой системе.
\end{abstract}

Ключевые слова: экситон, поляритон, сверхтекучесть, метод континуального интегрирования, приближение Попова.

\section{1. ВВЕДЕНИЕ}

В последнее время резко возрос интерес к бозе-конденсации системы композитных бозонов-экситонных поляритонов в оптической микрополости (см. работы [1]-[5] и библиографию в них). Вначале коротко опишем физическую модель. Рассматриваемая система представляет собой оптическую микрополость (прозрачную в изучаемой области пленку, ограниченную интерференционными зеркалами) с высокой добротностью. Внутрь микрополости погружена полупроводниковая квантовая яма. Дисперсионная зависимость фотонов в микрополости - зависимость частоты от продольного вектора $k_{\|}$- имеет при малых $k_{\|}$(существенных в области низких температур) следующий вид:

$$
\omega=c k=c \sqrt{\left(\frac{\pi n}{L}\right)^{2}+k_{\|}^{2}} \approx \omega_{n}(0)+\frac{k_{\|}^{2}}{2 m_{\mathrm{ph}}} .
$$

Таким образом, в условиях конфайнмента "квазидвумерный" фотон имеет эффективную продольную массу $m_{\mathrm{ph}}$, зависящую лишь от геометрических параметров микрополости (ее толщины $L$ ). Здесь $\omega_{n}(0)=\pi n c / L, c-$ скорость света в веществе

${ }^{*}$ Институт спектроскопии РАН, Троицк, Московская обл., Россия.

E-mail: lozovik@isan.troitsk.ru

${ }^{\dagger}$ Физический институт им. П. Н. Лебедева РАН, Москва, Россия. E-mail: semenov@lpi.ru 
микрополости, $m_{\mathrm{ph}}=\pi n /(L c)$; в дальнейшем для конкретности рассматривается нижняя поперечная мода $n=1$. Затухание этой моды, связанное с поглощением в оптической полости и выходом через полупрозрачные зеркала, ограничивающие полость, предполагается малым (высокая добротность полости) и в настоящей работе не учитывается.

В полупроводниковой квантовой яме, погруженной в полость, могут быть резонансно возбуждены фотонами микрополости квазидвумерные экситоны, которые при условии $n a^{* 2} \ll 1\left(n-\right.$ поверхностная концентрация экситонов, $a^{*}-$ их радиус) могут рассматриваться как бозоны. Они имеют дисперсионную зависимость $E\left(k_{\|}\right)=E_{0}+k_{\|}^{2} /\left(2 m_{\mathrm{ex}}\right)$. Таким образом, в системе имеются два типа взаимодействующих резонансным образом бозе-частиц. Резонансное взаимодействие двух типов бозонов обеспечивается специальным подбором геометрических параметров микрополости, при котором дисперсионные кривые взаимодействующих фотона в микрополости и экситона в квантовой яме пересекаются. В результате их антипересечения возникают квазидвумерные композитные бозонные квазичастицы - поляритоны в микрополости, представляющие собой суперпозицию квазидвумерных фотона микрополости и экситона. Однако результирующий закон дисперсии поляритонов полости (см. рис. 1) имеет сложный неквадратичный вид, а их эффективное взаимодействие оказывается зависящим от импульсов, что делает невозможным, например, применение обычного рецепта Ландау для вычисления сверхтекучей плотности в рассматриваемой системе (в противоположность системе экситонов, ср. с работами [6]).

Согласно общей теории в двумерной системе поляритонов при некоторой температуре должен происходить топологический переход Березинского-КостерлицаТаулеса в сверхтекучее состояние с алгебраическим недиагональным дальним порядком.

Интересной особенностью системы является то, что экситонный поляритон в микрополости может обладать малой эффективной массой $\left(10^{-4} m_{e}, m_{e}\right.$ - масса электрона), определяющейся в основном зависящим от геометрии оптической полости законом дисперсии фотона при малых продольных импульсах. Поэтому температура перехода, обратно пропорциональная эффективной массе, может достигать комнатных температур. Но после перехода системы поляритонов в фазу с квазидальним порядком фотоны, покидающие оптическую микрополость, должны обладать статистикой лазерного излучения. В этом смысле указанная система, рассматриваемая как источник излучения, является лазером, работающим без инверсии заселенностей.

В рассматриваемой системе из-за ее двумерности истинная бозе-конденсация отсутствует, и переход в сверхтекучее состояние, как уже говорилось, осуществляется посредством перехода Березинского-Костерлица-Таулеса [7]. Теория КостерлицаТаулеса использует в качестве первого этапа построение эффективного функционала, описывающего систему на больших масштабах. По причинам, указанным выше (неквадратичность затравочного закона дисперсии частиц и зависимость их взаимодействия от импульсов), необходимо такое обобщение аппарата, которое учитывало 


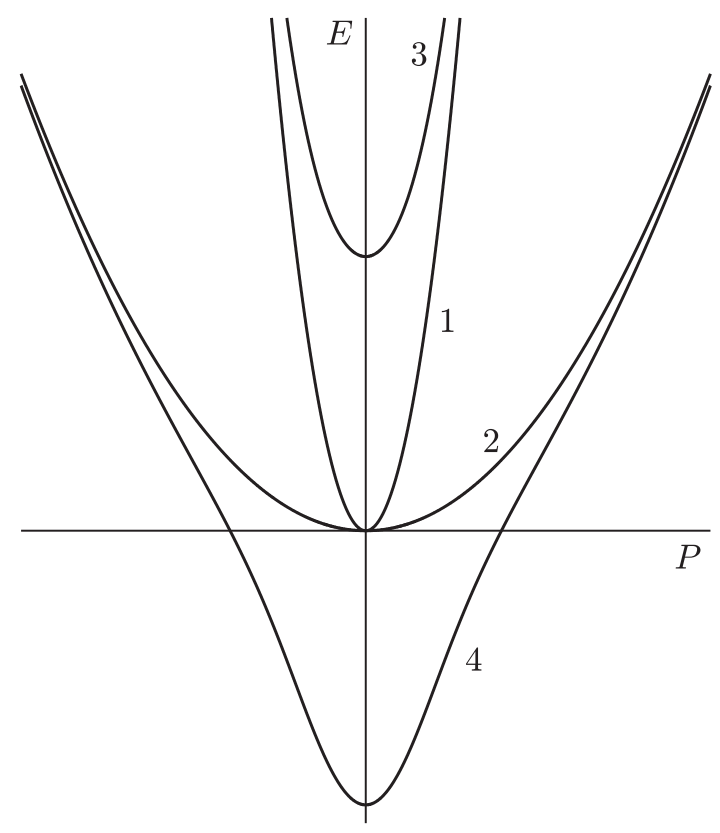

Рис. 1. Спектр экситонных поляритонов в оптической микрополости (в отсутствие поляритон-поляритонного взаимодействия): 1 - затравочный спектр фотона в полости; 2 - спектр экситона в квантовой яме; 3,4 - верхняя и нижняя ветви экситонных поляритонов в полости.

бы указанные особенности системы, что и является целью нашей работы. В первой части мы опишем общий способ получения эффективного действия, используя идеологию, предложенную Поповым [8]. Далее будет кратко описана поляритонная система и вычислена сверхтекучая плотность как функция температуры.

\section{2. НИЗКОЭНЕРГЕТИЧЕСКОЕ ЭФФЕКТИВНОЕ ДЕЙСТВИЕ}

Рассмотрим бозонную систему, статистическая сумма которой описывается стандартным образом через функциональный интеграл:

$$
Z=\int \mathcal{D} \bar{\psi} \mathcal{D} \psi \exp \left\{\int_{0}^{\beta} d \tau\left(\int \bar{\psi} \partial_{\tau} \psi-H_{\mathrm{p}}\right)\right\}
$$

где $H_{\mathrm{p}}$ - гамильтониан поляритонов. Будем действовать в духе подхода Попова [8]. Разделим флуктуирующее поле на медленную и быструю компоненты:

$$
\psi=\psi_{1}+\psi_{\mathrm{sh}},
$$

где в $\psi_{\mathrm{sh}}$ содержатся только фурье-компоненты с волновыми векторами больше некоторого $k_{\mathrm{c}}$, в то время как в $\psi_{1}$ - наоборот. Длинноволновую часть представим 
в переменных фаза-плотность и выделим из коротковолновой части фазу длинноволнового поля, т.е. представим компоненты поля в следующем виде:

$$
\psi_{\mathrm{l}}=\sqrt{n_{0}} e^{i \varphi} \quad \psi_{\mathrm{sh}}=\psi^{\prime} e^{i \varphi} .
$$

Проинтегрируем (1) по полям $\psi^{\prime}$, в результате чего получится эффективное действие, которое зависит от $\varphi$ и $n_{0}$. Основной вклад в длинноволновое поведение дают термодинамические флуктуации фазы. Воспользуемся следующим тождеством:

$$
\partial_{\mu}\left(e^{i \varphi(x)} f(x)\right)=e^{i \varphi(x)}\left(\partial_{\mu}+i\left(\partial_{\mu} \varphi(x)\right)\right) f(x),
$$

что формально соответствует использованию ковариантных производных вместо обычных. Тогда, используя вышеуказанную подстановку, получим, что система с фазой длинноволнового поля $\varphi$ соответствует бозе-конденсированной системе (в роли (квази)конденсата выступают частицы с импульсами меньше $k_{\mathrm{c}}$ ), взаимодействующей с векторным полем $A_{\mu}=\partial_{\mu} \varphi$. Поскольку мы рассматриваем длинноволновые флуктуации в системе и $A_{\mu}$ мало, то разложим действие до второго порядка по $A_{\mu}$ :

$$
S \approx S_{0}+\int d \tau d^{2} x J_{\mu}(x, \tau) A_{\mu}(x, \tau)+\frac{1}{2} \int d \tau d^{2} x d \tau^{\prime} d^{2} x^{\prime} \frac{\delta J_{\mu}(x, \tau)}{\delta A_{\nu}\left(x^{\prime}, \tau^{\prime}\right)}
$$

где $S_{0}$ - действие для бозе-системы с (квази)конденсатом и введено обозначение для тока

$$
J_{\mu}(x, \tau)=\frac{\delta S}{\delta A_{\mu}(x, \tau)} .
$$

Вычисляя средние, получим следующее выражение для эффективного действия:

$$
\begin{gathered}
S_{\mathrm{eff}}=\frac{1}{2} \int d \tau d^{2} x d \tau^{\prime} d^{2} x^{\prime} \Pi_{\mu \nu}\left(x, \tau, x^{\prime}, \tau^{\prime}\right) A_{\mu}(x, \tau) A_{\nu}\left(x^{\prime}, \tau^{\prime}\right), \\
\Pi_{\mu \nu}\left(x, \tau, x^{\prime}, \tau^{\prime}\right)=\left\langle\frac{\delta J_{\mu}(x, \tau)}{\delta A_{\nu}\left(x^{\prime}, \tau^{\prime}\right)}\right\rangle-\left\langle J_{\mu}(x, \tau) J_{\nu}\left(x^{\prime}, \tau^{\prime}\right)\right\rangle .
\end{gathered}
$$

После преобразования Фурье мы принимаем во внимание только термодинамические флуктуации, т.е. флуктуации с нулевой мацубаровской частотой:

$$
\Pi_{\mu \nu}\left(q, i \omega_{n}\right)=\left\langle K_{\mu \nu}\left(q, i \omega_{n}\right)\right\rangle-\left\langle J_{\mu}\left(q, i \omega_{n}\right) J_{\nu}\left(-q,-i \omega_{n}\right)\right\rangle,
$$

где мы ввели обозначение $K_{\mu \nu}=\delta J_{\mu} / \delta A_{\mu}$. В силу изотропии системы корреляционную функцию можно разложить на продольную и поперечную части. В пределе нулевого импульса корреляционная функция имеет вид

$$
\left\langle J_{\mu}(q, 0) J_{\nu}(-q, 0)\right\rangle=\chi_{l} \frac{q_{\mu} q_{\nu}}{q^{2}}+\chi_{t}\left(\delta_{\mu \nu}-\frac{q_{\mu} q_{\nu}}{q^{2}}\right), \quad q \rightarrow 0 .
$$

Далее будет показано при помощи правила сумм, что в пределе нулевых импульсов

$$
K_{\mu \nu}(0,0)=\chi_{l} \delta_{\mu \nu}
$$


Таким образом, при малых импульсах получаем следующее выражение:

$$
\Pi_{\mu \nu}(q, 0)=\left(\chi_{l}-\chi_{t}\right)\left(\delta_{\mu \nu}-\frac{q_{\mu} q_{\nu}}{q^{2}}\right), \quad q \rightarrow 0 .
$$

Нетрудно видеть, что получился чисто поперечный тензор. Может показаться, что в силу определения векторное поле $A_{\mu}$ является чисто продольным и результирующее действие равно нулю, однако это не так, если мы рассматриваем возбуждения типа квантовых вихрей. Именно поэтому действие отлично от нуля только при наличии вихрей. При этом сверхтекучая плотность равна

$$
\rho_{\mathrm{s}}=\chi_{l}-\chi_{t}
$$

Отсюда результирующее действие имеет стандартный вид:

$$
S_{\mathrm{eff}}=\frac{\rho_{\mathrm{s}}}{2 T} \int d^{2} x\left(\partial_{\mu} \varphi_{\mathrm{v}}(x)\right)^{2}
$$

где $\varphi_{\mathrm{v}}(x)$ - вихревая составляющая фазы $\varphi(x)$. Таким образом, интересной для изучения в данной системе величиной является разность продольной и поперечной восприимчивостей.

Перейдем теперь к описанию интересующей нас системы поляритонов.

\section{3. ОПЕРАТОР ТОКА В ПОЛЯРИТОННОЙ СИСТЕМЕ}

Описанное в предыдущем разделе вычисление довольно общее, и его можно применять к любой бозе-системе. Для нас интерес представляет система поляритонов в двумерной микрополости, состоящая из слабо взаимодействующих экситонов и невзаимодействующих фотонов, при этом фотоны и экситоны могут взаимно превращатся друг в друга. При отсутствии взаимодействия между экситонами нормальными возбуждениями в данной системе являются нижний и верхний поляритоны, закон дисперсии которых имеет следующий вид (см. рис. 1):

$$
\mathcal{E}_{l, u}\left(p^{2}\right)=\frac{1}{2}\left(\frac{p^{2}}{2 m_{\mathrm{ph}}}+\frac{p^{2}}{2 m_{\mathrm{ex}}}\right) \pm \frac{1}{2} \sqrt{\left(\frac{p^{2}}{2 m_{\mathrm{ph}}}-\frac{p^{2}}{2 m_{\mathrm{ex}}}\right)^{2}+\Omega^{2}},
$$

где $\Omega$ - поляритонное расщепление, а $m_{\mathrm{ex}}$ и $m_{\mathrm{ph}}-$ экситонная и фотонная массы соответственно. Еще раз отметим, что под фотонной массой мы понимаем эффективную массу, которая возникает оттого, что в направлении, перепендикулярном полости, волновой вектор квантуется, и зависимость энергии фотона от продольного импульса имеет квадратичный вид при малых импульсах. Данная масса определяется лишь геометрическими свойствами полости.

При низких температурах заселяется преимущественно нижняя поляритонная ветвь, поэтому мы будем рассматривать далее только ее, считая верхнюю ветвь 
незаселенной. При учете экситон-экситонного взаимодействия гамильтониан системы и соответствующее действие примут следующий вид:

$$
\begin{aligned}
\widehat{H}=\sum_{p} \mathcal{E}\left(p^{2}\right) \psi_{p}^{\dagger} \psi_{p}+\frac{g}{2} \sum_{p_{1}+p_{2}=p_{3}+p_{4}} \mathcal{X}\left(p_{1}^{2}\right) \mathcal{X}\left(p_{2}^{2}\right) \mathcal{X}\left(p_{3}^{2}\right) \mathcal{X}\left(p_{4}^{2}\right) \psi_{p_{1}}^{\dagger} \psi_{p_{2}}^{\dagger} \psi_{p_{3}} \psi_{p_{4}}, \\
S=\int d \tau\left(\sum_{p} \bar{\psi}_{p}\left(\partial_{\tau}-\mathcal{E}\left(p^{2}\right)+\mu\right) \psi_{p}-\right. \\
\left.\quad-\frac{g}{2} \sum_{p_{1}+p_{2}=p_{3}+p_{4}} \mathcal{X}\left(p_{1}^{2}\right) \mathcal{X}\left(p_{2}^{2}\right) \mathcal{X}\left(p_{3}^{2}\right) \mathcal{X}\left(p_{4}^{2}\right) \bar{\psi}_{p_{1}} \bar{\psi}_{p_{2}} \psi_{p_{3}} \psi_{p_{4}}\right)
\end{aligned}
$$

где $g$ - константа экситон-экситонного взаимодействия, а $\mathcal{X}\left(p^{2}\right)$ - так называемый коэффициент Хопфилда, который описывает смешивание экситонной и фотонной ветвей и равен

$$
\mathcal{X}\left(p^{2}\right)=\sqrt{\frac{1}{2}+\frac{p^{2} /\left(2 m_{\mathrm{ph}}\right)-p^{2} /\left(2 m_{\mathrm{ex}}\right)}{2 \sqrt{\left(p^{2} /\left(2 m_{\mathrm{ph}}\right)-p^{2} /\left(2 m_{e x}\right)\right)^{2}+\Omega^{2}}}} .
$$

Система с данным гамильтонианом отличается от обычной бозе-системы неквадратичным законом дисперсии и специфическим взаимодействием, матричный элемент которого зависит от всех четырех импульсов, в то время как обычно он зависит лишь от переданного импульса. Поэтому оператор тока будет отличен от известного выражения. Как было указано в предыдущем разделе, для получения выражения для тока необходимо проварьировать действие по векторному потенциалу. Используя метод, описанный в приложении, получим следующее выражение:

$$
J_{q}^{\mu}=\sum_{p_{1}, p_{2}} J_{1}^{\mu}\left(p_{1} ; p_{2} \mid q\right) \bar{\psi}_{p_{1}} \psi_{p_{2}}+\sum_{p_{1}, p_{2}, p_{3}, p_{4}} J_{2}^{\mu}\left(p_{1}, p_{2} ; p_{3}, p_{4} \mid q\right) \bar{\psi}_{p_{1}} \bar{\psi}_{p_{2}} \psi_{p_{3}} \psi_{p_{4}},
$$

где введены следующие обозначения для вершин:

$$
\begin{aligned}
& J_{1}^{\mu}\left(p_{1} ; p_{2} \mid q\right)= \widetilde{E}_{q, p_{2}}^{\mu} \delta_{p_{1}, p_{2}+q}=\left(p_{1 \mu}+p_{2 \mu}\right) \frac{\mathcal{E}\left(p_{1}^{2}\right)-\mathcal{E}\left(p_{2}^{2}\right)}{p_{1}^{2}-p_{2}^{2}} \delta_{p_{1}, p_{2}+q}, \\
& J_{2}^{\mu}\left(p_{1}, p_{2} ; p_{3}, p_{4} \mid q\right)=\frac{g}{2} X_{-q, p_{1}}^{\mu} \mathcal{X}\left(p_{2}^{2}\right) \mathcal{X}\left(p_{3}^{2}\right) \mathcal{X}\left(p_{4}^{2}\right) \delta_{p_{1}+p_{2}-q, p_{3}+p_{4}}+ \\
&+ \frac{g}{2} \mathcal{X}\left(p_{1}^{2}\right) X_{-q, p_{2}}^{\mu} \mathcal{X}\left(p_{3}^{2}\right) \mathcal{X}\left(p_{4}^{2}\right) \delta_{p_{1}+p_{2}-q, p_{3}+p_{4}}+ \\
&+ \frac{g}{2} \mathcal{X}\left(p_{1}^{2}\right) \mathcal{X}\left(p_{2}^{2}\right) X_{q, p_{3}}^{\mu} \mathcal{X}\left(p_{4}^{2}\right) \delta_{p_{1}+p_{2}, p_{3}+p_{4}+q}+ \\
&+\frac{g}{2} \mathcal{X}\left(p_{1}^{2}\right) \mathcal{X}\left(p_{2}^{2}\right) \mathcal{X}\left(p_{3}^{2}\right) X_{q, p_{4}}^{\mu} \delta_{p_{1}+p_{2}, p_{3}+p_{4}+q} \\
& X_{q, p}^{\mu}=\left(2 p_{\mu}+q_{\mu}\right) \frac{\mathcal{X}\left((p+q)^{2}\right)-\mathcal{X}\left(p^{2}\right)}{(p+q)^{2}-p^{2}}
\end{aligned}
$$

\section{4. ПРОДОЛЬНАЯ ВОСПРИИМЧИВОСТЬ}

В предыдущем разделе было получено выражение для тока. Теперь можно уже вычислять корреляционную функцию токов, однако, как известно, при использовании приближения Боголюбова продольная восприимчивость вычисляется в древесном приближении, в то время как поперечная - в однопетлевом. Поэтому для 
вычисления поперечной восприимчивости удобно воспользоваться правилом сумм, согласно которому (см. например, книгу [9])

$$
\chi_{l}(q, 0)=\frac{1}{q^{2}}\left\langle\left[\hat{n}_{q},\left[\widehat{H}, \hat{n}_{-q}\right]\right]\right\rangle .
$$

Оператор плотности числа частиц имеет вид

$$
\hat{n}_{q}=\sum_{p} \psi_{p+q}^{\dagger} \psi_{p}
$$

В данное правило сумм вклад будут давать оба члена гамильтониана. Первый вклад вычисляется несложно и равен

$$
\chi_{\mathrm{L}}^{(1)}(q, 0)=\frac{1}{q^{2}} \sum_{p}\left(\mathcal{E}\left((p+q)^{2}\right)+\mathcal{E}\left((p-q)^{2}\right)-2 \mathcal{E}\left(p^{2}\right)\right)\left\langle\psi_{p}^{\dagger} \psi_{p}\right\rangle .
$$

При импульсе, стремящемся к нулю, данное выражение превращается в следующее (здесь и далее размерность пространства $d=2$ ):

$$
\chi_{\mathrm{L}}^{(1)}(0,0)=\sum_{p}\left(2 \mathcal{E}^{\prime}\left(p^{2}\right)+\frac{4}{d} p^{2} \mathcal{E}^{\prime \prime}\left(p^{2}\right)\right)\left\langle\psi_{p}^{\dagger} \psi_{p}\right\rangle .
$$

Несложно видеть, что данное выражение есть среднее от эффективного тензора массы, который определяется как

$$
\frac{1}{m_{i j}^{*}}=\frac{\partial^{2} \mathcal{E}\left(p^{2}\right)}{\partial p_{i} \partial p_{j}}
$$

Вторая часть тока более сложная. Она имеет вид

$$
\chi_{\mathrm{L}}^{(2)}(q, 0)=\frac{g}{q^{2}} \sum_{p_{1}+p_{2}=p_{3}+p_{4}} \Upsilon\left(p_{1}, p_{2} ; p_{3}, p_{4} \mid q\right)\left\langle\psi_{p_{1}}^{\dagger} \psi_{p_{2}}^{\dagger} \psi_{p_{3}} \psi_{p_{4}}\right\rangle,
$$

где мы ввели обозначение

$$
\begin{aligned}
\Upsilon\left(p_{1},\right. & \left.p_{2} ; p_{3}, p_{4} \mid q\right)=2 \mathcal{X}\left(\left(p_{1}+q\right)^{2}\right) \mathcal{X}\left(\left(p_{3}+q\right)^{2}\right)+ \\
& +2 \mathcal{X}\left(\left(p_{1}-q\right)^{2}\right) \mathcal{X}\left(\left(p_{3}-q\right)^{2}\right)-2 \mathcal{X}\left(p_{1}^{2}\right) \mathcal{X}\left(p_{3}^{2}\right) \mathcal{X}\left(p_{2}^{2}\right) \mathcal{X}\left(p_{4}^{2}\right)- \\
& -\mathcal{X}\left(\left(p_{1}+q\right)^{2}\right) \mathcal{X}\left(\left(p_{2}-q\right)^{2}\right) \mathcal{X}\left(p_{3}^{2}\right) \mathcal{X}\left(p_{4}^{2}\right)- \\
& -\mathcal{X}\left(p_{1}^{2}\right) \mathcal{X}\left(p_{2}^{2}\right) \mathcal{X}\left(\left(p_{3}+q\right)^{2}\right) \mathcal{X}\left(\left(p_{4}-q\right)^{2}\right) .
\end{aligned}
$$

Нетрудно видеть, что при $q \rightarrow 0$ это выражение принимает вид

$$
\begin{aligned}
& \lim _{q \rightarrow 0} \frac{\Upsilon\left(p_{1}, p_{2} ; p_{3}, p_{4} \mid q\right)}{q^{2}}= \\
& \quad=\lim _{q \rightarrow 0} \frac{q_{\mu} q_{\nu}}{2 q^{2}} \frac{\partial^{2}}{\partial q_{\mu} \partial q_{\nu}}\left(\mathcal{X}\left(\left(p_{1}+q\right)^{2}\right) \mathcal{X}\left(\left(p_{2}+q\right)^{2}\right) \mathcal{X}\left(\left(p_{3}+q\right)^{2}\right) \mathcal{X}\left(\left(p_{4}+q\right)^{2}\right)\right)
\end{aligned}
$$

и в совокупности с $(25)$ дает $\left\langle K_{\mu \nu}(0,0)\right\rangle$, благодаря чему мы и получили поперечный отклик. Таким образом, используя правило сумм, мы выразили продольную восприимчивость через одночастичные средние. Теперь можно приступать к вычислению данных корреляторов в рамках теории слабо неидеального бозе-газа. 


\section{5. СЛАБО НЕИДЕАЛЬНЫЙ БОЗЕ-ГАЗ ПОЛЯРИТОНОВ}

Прежде чем перейти к непосредственному вычислению функции отклика, выпишем основные соотношения, касающиеся бозе-газа. Более подробно микроскопическая теория данной системы будет описана в другой статье. Мы будем рассматривать двумерный случай. В соответствии с теорией Боголюбова выделяется конденсат, роль которого играют частицы с импульсами, меньшими $k_{\mathrm{c}}$. В этом случае плотность частиц и химический потенциал в однопетлевом приближении имеют следующий вид:

$$
\begin{gathered}
n=n_{0}+\frac{1}{2 V} \sum_{|p|>k_{\mathrm{c}}}\left(\frac{\varepsilon_{p}+2 G_{p} n_{0}-\mu}{\varepsilon_{\mathrm{b}}}\left(2 n_{\mathrm{b}}\left(\varepsilon_{\mathrm{b}}\right)+1\right)-1\right), \\
\mu=G_{0} n_{0}+\frac{1}{V} \sum_{|p|>k_{\mathrm{c}}} G_{p}\left(\frac{2 \varepsilon_{p}+3 G_{p} n_{0}-2 \mu}{2 \varepsilon_{\mathrm{b}}}\left(2 n_{\mathrm{b}}\left(\varepsilon_{\mathrm{b}}\right)+1\right)-1\right), \\
\varepsilon_{p} \equiv \mathcal{E}\left(p^{2}\right), \quad X_{p} \equiv \mathcal{X}\left(p^{2}\right), \quad \varepsilon_{\mathrm{b}}=\sqrt{\left(\varepsilon_{p}+2 G_{p} n_{0}-\mu\right)^{2}-G_{p}^{2} n_{0}^{2}}, \\
G_{p}=g \mathcal{X}\left(p^{2}\right) \mathcal{X}\left(p^{2}\right) \mathcal{X}(0) \mathcal{X}(0), \quad n_{\mathrm{b}}(\varepsilon)=\frac{1}{e^{\varepsilon / T}-1} .
\end{gathered}
$$

Отсюда следует уравнение состояния

$$
\begin{aligned}
n=\frac{\mu}{G_{0}} & +\frac{1}{V} \sum_{p} \frac{2 G_{p}-G_{0}}{2 G_{0}^{2}}+ \\
& +\frac{1}{V} \sum_{p} \frac{G_{0}\left(G_{0}-2 G_{p}\right) \varepsilon_{p}+\mu\left(4 G_{p} G_{0}-G_{0}^{2}-3 G_{p}^{2}\right)}{2 \varepsilon_{\mathrm{b}} G_{0}^{2}}\left(2 n_{\mathrm{b}}\left(\varepsilon_{\mathrm{b}}\right)+1\right) .
\end{aligned}
$$

Данное уравнение, однако, содержит ультрафиолетовую расходимость, которая устраняется перенормировкой константы связи и соответствует суммированию лестничных диаграмм. В этом случае константа связи заменяется на вершину Г. В результате уравнение состояния приобретает вид

$$
\begin{gathered}
n=\frac{\mu}{\Gamma}+\frac{1}{V} \sum_{p}\left(\left(1-\frac{2 X_{p}^{2}}{X_{0}^{2}}\right) \frac{\varepsilon_{p}}{\varepsilon_{\mathrm{b}}}+\left(\frac{4 X_{p}^{2}}{X_{0}^{2}}-\frac{3 X_{p}^{4}}{X_{0}^{4}}-1\right) \frac{\mu}{\varepsilon_{\mathrm{b}}}\right) n_{\mathrm{b}}\left(\varepsilon_{\mathrm{b}}\right), \\
\varepsilon_{\mathrm{b}}=\sqrt{\left(\varepsilon_{p}+\mu \frac{2 X_{p}^{2}-X_{0}^{2}}{X_{0}^{2}}\right)^{2}-\frac{X_{p}^{4} \mu^{2}}{X_{0}^{4}}} .
\end{gathered}
$$

Также нам понадобятся нормальная и аномальная функции Грина. В мацубаровском представлении они имеют вид

$$
\begin{gathered}
\mathcal{G}(p, n)=-\left\langle\bar{\psi}_{p, n} \psi_{p, n}\right\rangle=-\frac{i \omega_{n}+\varepsilon_{p}-\mu+2 G_{p} n_{0}}{\omega_{n}^{2}+\varepsilon_{\mathrm{b}}^{2}}, \\
\mathcal{G}_{\mathrm{a}}(p, n)=-\left\langle\psi_{p, n} \psi_{-p,-n}\right\rangle=\frac{G_{p} n_{0}}{\omega_{n}^{2}+\varepsilon_{\mathrm{b}}^{2}} .
\end{gathered}
$$

Перейдем теперь к вычислению восприимчивостей в пределе нулевых импульсов в данной модели. 


\section{6. ПРОДОЛЬНАЯ И ПОПЕРЕЧНАЯ ВОСПРИИМЧИВОСТИ СЛАБО НЕИДЕАЛЬНОГО БОЗЕ-ГАЗА ПОЛЯРИТОНОВ}

Как уже говорилось, для вычисления продольной восприимчивости будем использовать правило сумм. Как было показано в предыдущем разделе, продольная восприимчивость состоит из двух частей. Первая часть имеет вид

$$
\begin{aligned}
\chi_{\mathrm{L}}^{(1)}(0,0) & =2 n_{0} \mathcal{E}^{\prime}(0)+ \\
+ & \frac{1}{V} \sum_{p}\left(\mathcal{E}^{\prime}\left(p^{2}\right)+\frac{2}{d} p^{2} \mathcal{E}^{\prime \prime}\left(p^{2}\right)\right)\left(\frac{\varepsilon_{p}+2 G_{p} n_{0}-\mu}{\varepsilon_{\mathrm{b}}}\left(2 n_{\mathrm{b}}\left(\varepsilon_{\mathrm{b}}\right)+1\right)-1\right) .
\end{aligned}
$$

От имеющейся здесь инфракрасной расходимости избавимся, выразив плотность конденсата через полную плотность. В результате выражение запишем как

$$
\begin{aligned}
\chi_{\mathrm{L}}^{(1)}(0,0) & =2 n \mathcal{E}^{\prime}(0)+ \\
+ & \frac{1}{V} \sum_{p}\left(\mathcal{E}^{\prime}\left(p^{2}\right)-\mathcal{E}^{\prime}(0)+\frac{2}{d} p^{2} \mathcal{E}^{\prime \prime}\left(p^{2}\right)\right)\left(\frac{\varepsilon_{p}}{\varepsilon_{\mathrm{b}}}+\left(\frac{2 X_{p}^{2}}{X_{0}^{2}}-1\right) \frac{\mu}{\varepsilon_{\mathrm{b}}}-1\right)+ \\
+ & \frac{2}{V} \sum_{p}\left(\mathcal{E}^{\prime}\left(p^{2}\right)-\mathcal{E}^{\prime}(0)+\frac{2}{d} p^{2} \mathcal{E}^{\prime \prime}\left(p^{2}\right)\right)\left(\frac{\varepsilon_{p}}{\varepsilon_{\mathrm{b}}}+\left(\frac{2 X_{p}^{2}}{X_{0}^{2}}-1\right) \frac{\mu}{\varepsilon_{\mathrm{b}}}\right) n_{\mathrm{b}}\left(\varepsilon_{\mathrm{b}}\right) .
\end{aligned}
$$

Полученное выражение есть однопетлевой результат. В соответствии с этим и все остальные части отклика будем вычислять в том же приближении. Тогда в среднем от четырех операторов рождения и уничтожения, которое входит во вторую часть продольного отклика, необходимо оставить после боголюбовского выделения конденсата только квадратичную часть. Таким образом, имеем

$$
\begin{aligned}
& \chi_{\mathrm{L}}^{(2)}(0,0)=4 g n_{0}^{2} \mathcal{X}^{3}(0) \mathcal{X}^{\prime}(0)+\frac{4 g n_{0}}{d V} \sum_{p} p^{2} \mathcal{A}_{p} \mathcal{X}^{2}(0) \mathcal{X}^{\prime 2}\left(p^{2}\right)+ \\
& \quad+\frac{g n_{0}}{V} \sum_{p} \mathcal{B}_{p} \mathcal{X}\left(p^{2}\right) \mathcal{X}(0)\left(2 \mathcal{X}\left(p^{2}\right) \mathcal{X}^{\prime}(0)+2 \mathcal{X}(0) \mathcal{X}^{\prime}\left(p^{2}\right)+\frac{4}{d} p^{2} \mathcal{X}(0) \mathcal{X}^{\prime \prime}\left(p^{2}\right)\right)
\end{aligned}
$$

где

$$
\begin{aligned}
& \mathcal{A}_{p}=\frac{4 \varepsilon_{p}+10 G_{p} n_{0}-4 \mu}{2 \varepsilon_{\mathrm{b}}}\left(2 n_{\mathrm{b}}\left(\varepsilon_{\mathrm{b}}\right)+1\right)-2, \\
& \mathcal{B}_{p}=\frac{4 \varepsilon_{p}+6 G_{p} n_{0}-4 \mu}{2 \varepsilon_{\mathrm{b}}}\left(2 n_{\mathrm{b}}\left(\varepsilon_{\mathrm{b}}\right)+1\right)-2 .
\end{aligned}
$$

Аналогично предыдущему случаю, избавляемся от расходимостей, выражая $g n_{0}^{2}$ через $\mu n$ в однопетлевом приближении:

$$
\begin{aligned}
\mu n=G_{0} & n_{0}^{2}-\frac{n_{0}}{V} \sum_{p} \frac{G_{0}+2 G_{p}}{2}+ \\
& +\frac{n_{0}}{V} \sum_{p} \frac{\left(\varepsilon_{p}-\mu\right)\left(G_{0}+2 G_{p}\right)+G_{p} n_{0}\left(2 G_{0}+3 G_{p}\right)}{2 \varepsilon_{\mathrm{b}}}\left(2 n_{\mathrm{b}}\left(\varepsilon_{\mathrm{b}}\right)+1\right) .
\end{aligned}
$$


В результате получится выражение, которое будет свободно от расходимостей:

$$
\begin{aligned}
\chi_{\mathrm{L}}^{(2)}(0,0)=4 & \mu \frac{\mathcal{X}^{\prime}(0)}{\mathcal{X}(0)}+ \\
& +\frac{2 \mu}{X_{0}^{2} V} \sum_{p} X_{p}\left(\mathcal{X}^{\prime}\left(p^{2}\right)+\frac{2}{d} p^{2} \mathcal{X}^{\prime \prime}\left(p^{2}\right)\right)\left(2 \frac{\varepsilon_{p}}{\varepsilon_{\mathrm{b}}}+\left(3 \frac{X_{p}^{2}}{X_{0}^{2}}-2\right) \frac{\mu}{\varepsilon_{\mathrm{b}}}-2\right)+ \\
& +\frac{4 \mu}{X_{0}^{2} V} \sum_{p} X_{p}\left(\mathcal{X}^{\prime}\left(p^{2}\right)+\frac{2}{d} p^{2} \mathcal{X}^{\prime \prime}\left(p^{2}\right)\right)\left(2 \frac{\varepsilon_{p}}{\varepsilon_{\mathrm{b}}}+\left(3 \frac{X_{p}^{2}}{X_{0}^{2}}-2\right) \frac{\mu}{\varepsilon_{\mathrm{b}}}\right) n_{\mathrm{b}}\left(\varepsilon_{\mathrm{b}}\right)+ \\
& +\frac{4 \mu}{d X_{0}^{2} V} \sum_{p} p^{2}\left(\mathcal{X}^{\prime}\left(p^{2}\right)\right)^{2}\left(2 \frac{\varepsilon_{p}}{\varepsilon_{\mathrm{b}}}+\left(5 \frac{X_{p}^{2}}{X_{0}^{2}}-2\right) \frac{\mu}{\varepsilon_{\mathrm{b}}}-2\right)+ \\
& +\frac{8 \mu}{d X_{0}^{2} V} \sum_{p} p^{2}\left(\mathcal{X}^{\prime}\left(p^{2}\right)\right)^{2}\left(2 \frac{\varepsilon_{p}}{\varepsilon_{\mathrm{b}}}+\left(5 \frac{X_{p}^{2}}{X_{0}^{2}}-2\right) \frac{\mu}{\varepsilon_{\mathrm{b}}}\right) n_{\mathrm{b}}\left(\varepsilon_{\mathrm{b}}\right)- \\
& -\frac{2 \mu \mathcal{X}^{\prime}(0)}{X_{0} V} \sum_{p}\left(\frac{\varepsilon_{p}}{\varepsilon_{\mathrm{b}}}+\left(2 \frac{X_{p}^{2}}{X_{0}^{2}}-1\right) \frac{\mu}{\varepsilon_{\mathrm{b}}}-1\right)- \\
& -\frac{4 \mu \mathcal{X}^{\prime}(0)}{X_{0} V} \sum_{p}\left(\frac{\varepsilon_{p}}{\varepsilon_{\mathrm{b}}}+\left(2 \frac{X_{p}^{2}}{X_{0}^{2}}-1\right) \frac{\mu}{\varepsilon_{\mathrm{b}}}\right) n_{\mathrm{b}}\left(\varepsilon_{\mathrm{b}}\right) .
\end{aligned}
$$

Сумма данного выражения и выражения (40) дает ответ для продольной восприимчивости слабо неидеального бозе-газа поляритонов.

Поперечная восприимчивость вычисляется стандартным образом через корреляционную функцию ток-ток. Для того чтобы вычислить ее в однопетлевом приближении, необходимо разложить оператор тока с точностью до двух операторов рождения-уничтожения надконденсатных частиц. В результате вклад дают диаграммы типа изображенных на рис. 2.

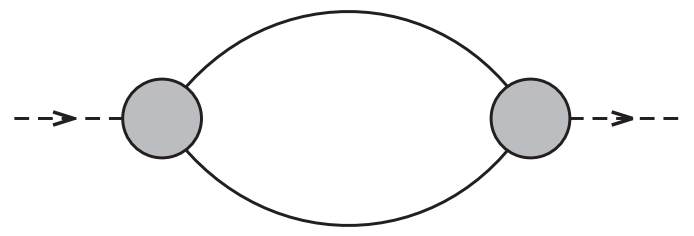

Рис. 2. Диаграммы для коррелятора ток-ток; на линиях необходимо расставить стрелки всевозможными способами; кружок обозначает токовую вершину.

Суммирование по мацубаровским частотам производится стандартным образом и дает прозводную от распределения Бозе. В результате получим следующее выражение для поперечной восприимчивости:

$$
\chi_{\mathrm{T}}(0,0)=-\frac{4}{d V} \sum_{p} p^{2}\left(\mathcal{E}^{\prime}\left(p^{2}\right)+4 \mu \mathcal{X}^{-2}(0) \mathcal{X}\left(p^{2}\right) \mathcal{X}^{\prime}\left(p^{2}\right)\right)^{2} n_{\mathrm{b}}^{\prime}\left(\varepsilon_{\mathrm{b}}\right) .
$$


Таким образом, нами были рассчитаны продольная и поперечная восприимчивости в приближении Боголюбова. С помощью этих выражений мы вычислили сверхтекучую плотность как функцию температуры при различных значениях перенормированной константы связи Г. На рис. 3 изображена зависимость “сверхтекучей плотности" от температуры при разных значениях Г. Интересной особенностью является то, что при некоторых значениях перенормированной константы связи сверхтекучая плотность с ростом температуры сначала растет и только потом уменьшается, что обусловлено лишь зависимостью взаимодействия от скорости. Подчеркнем, однако, что этот эффект может быть артефактом выбранного приближения.

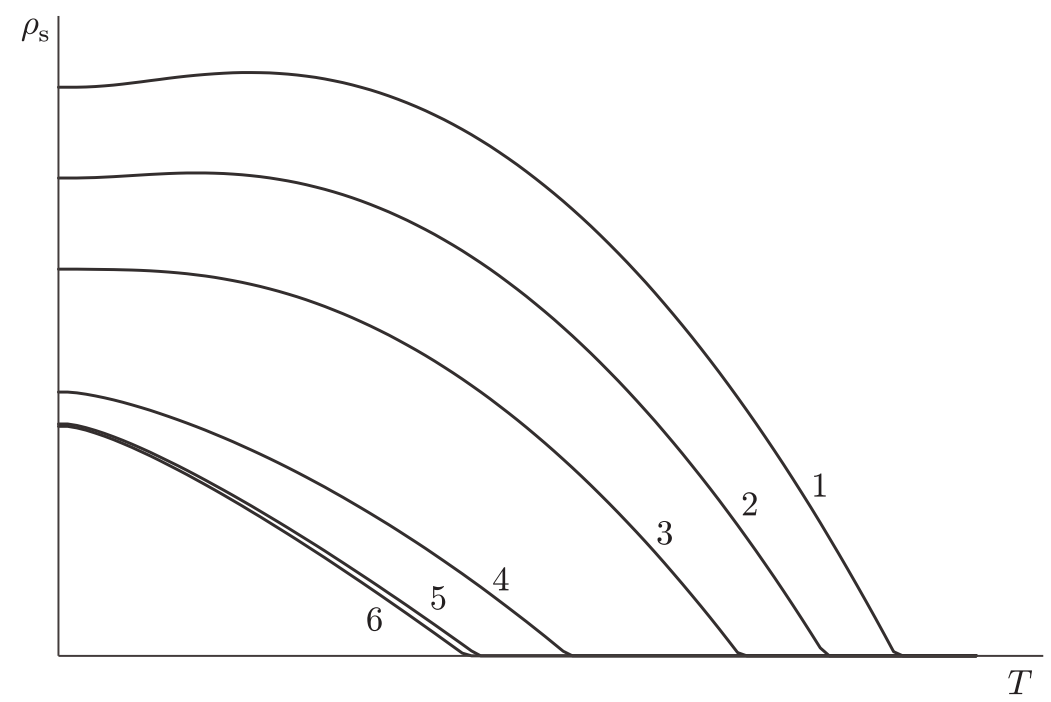

Рис. 3. Зависимость сверхтекучей плотности от температуры при разных значения константы связи $Г$ : кривая $1-\Gamma=1.3 \cdot 10^{-12}$ мэВ см $^{2}$, кривая $2-\Gamma=1.0 \cdot 10^{-12}$ мэВ $\mathrm{cm}^{2}$, кривая $3-\Gamma=6.0 \cdot 10^{-13}$ мэВ $\mathrm{cm}^{2}$, кривая $4-\Gamma=1.3 \cdot 10^{-13}$ мэВ·см ${ }^{2}$, кривая $5-\Gamma=1.3 \cdot 10^{-14}$ мэВ $\mathrm{cm}^{2}$, кривая $6-$ $\Gamma=1.3 \cdot 10^{-15}$ мэВ $\mathrm{cm}^{2}$. Плотность $n=1.5 \cdot 10^{13} \mathrm{~cm}^{-2}$, величина поляритонного расщепления $\Omega=26$ мэВ.

В рассматриваемой системе происходит переход Березинского-Костерлица-Таулеса [7] при температуре $T_{\mathrm{BKT}}=(\pi / 2) \rho_{\mathrm{s}}(T)$, где $\rho_{\mathrm{s}}(T)$ - определенная нами зависимость сверхтекучей плотности от температуры, приведенная на рис. 3. В точке перехода $T_{\mathrm{BKT}}$, как известно [10], происходит универсальный скачок сверхтекучей плотности до нуля.

\section{7. ЗАКЛЮЧЕНИЕ}

В данной работе было получено общее выражение для эффективного низкоэнергетического действия системы бозонов с произвольным затравочным законом дисперсии. Показано, что эффективное действие выражается через корреляционную функцию токов. Были получены выражение для тока для системы поляритонов 
и правило сумм для корреляционной функции токов. Корреляционные функции токов были вычислены в рамках приближения Боголюбова. В этом приближении найдена зависимость сверхтекучей плотности от температуры.

\section{ПРИЛОЖЕНИЕ}

Расмотрим полевой оператор $\hat{\psi}(x)$ и подействуем на него оператором $\widehat{B}$. Пусть этот оператор есть функция квадрата оператора импульса, т.е.

$$
\widehat{B}=\mathcal{B}\left(-\partial_{\mu}^{2}\right)=\sum_{n} c_{n}\left(-\partial_{\mu}^{2}\right)^{n} .
$$

В импульсном представлении имеем следующие простые соотношения:

$$
\widehat{B} \psi(x)=\widehat{B}\left(\sum_{p} \psi_{p} e^{i p x}\right)=\sum_{p} \mathcal{B}\left(p^{2}\right) \psi_{p} e^{i p x} .
$$

Выясним теперь, как изменяется данное выражение, если обычную производную заменить на ковариантную:

$$
\begin{gathered}
\partial_{\mu} \longrightarrow D_{\mu}=\partial_{\mu}+i A_{\mu}(x)=\partial_{\mu}+i \sum_{q} A_{q}^{\mu} e^{i q x}, \\
\widehat{B} \longrightarrow \widehat{B}^{\prime}=\mathcal{B}\left(-D_{\mu}^{2}\right) .
\end{gathered}
$$

Вычислим член, линейный по $A_{\mu}$. Нетрудно получить, что с точностью до линейного по $A_{\mu}$ члена

$$
\begin{aligned}
& -\left(\partial_{\mu}+i \sum_{q_{1}} A_{q_{1}}^{\mu} e^{i q_{1} x}\right)\left(\partial_{\mu}+i \sum_{q_{2}} A_{q_{2}}^{\mu} e^{i q_{2} x}\right) e^{i p x}= \\
& =p^{2} e^{i p x}+\sum_{q}\left(p_{\mu}+\left(p_{\mu}+q_{\mu}\right)\right) A_{q}^{\mu} e^{i(p+q) x}+O\left(A^{2}\right) .
\end{aligned}
$$

Используя это выражение, получим следующее равенство:

$$
\begin{aligned}
\widehat{B}^{\prime} e^{i p x} & =\mathcal{B}\left(p^{2}\right) e^{i p x}+\sum_{q} A_{q}^{\mu} \widetilde{B}_{q, p}^{\mu} e^{i(p+q) x}+O\left(A^{2}\right), \\
\widetilde{B}_{q, p}^{\mu} & =\sum_{n} c_{n} \sum_{k=1}^{n}(p+q)^{2(k-1)}\left(p_{\mu}+\left(p_{\mu}+q_{\mu}\right)\right) p^{2(n-k)}= \\
& =\left(2 p_{\mu}+q_{\mu}\right) \frac{\mathcal{B}\left((p+q)^{2}\right)-\mathcal{B}\left(p^{2}\right)}{(p+q)^{2}-p^{2}} .
\end{aligned}
$$

Таким образом, получаем

$$
\widehat{B}^{\prime} \psi(x)=\widehat{B} \psi(x)+\sum_{p, q} \psi_{p} A_{q}^{\mu} \widetilde{B}_{q, p}^{\mu} e^{i(p+q) x}+O\left(A^{2}\right) .
$$

Аналогично для комплексно-сопряженной величины

$$
\widehat{\widehat{B}^{\prime} \psi(x)}=\widehat{B} \bar{\psi}(x)+\sum_{p, q} \bar{\psi}_{p} A_{q}^{\mu} \widetilde{B}_{-q, p}^{\mu} e^{i(q-p) x}+O\left(A^{2}\right) .
$$


Благодарности. Работа поддержана РФФИ (грант № 05-02-17872). Один из авторов (А. Г. Семенов) поддержан фондом “Династия", УНК ФИАН и Целевой программой Президиума РАН поддержки молодых ученых.

\section{Список литературы}

[1] A. Kavokin, G. Malpuech, Cavity Polaritons, Elsevier, Amsterdam, 2003.

[2] H. Deng, G. Weihs, C. Santori, J. Bloch, Y. Yamamoto, Science, 298 (2002), 199.

[3] H. Cao, S. Pau, J. M. Jacobson, G. Bjork, Y. Yamamoto, A. Imamŏglu, Phys. Rev. A, 55 (1997), 4632.

[4] Ю.Е. Лозовик, А. Г. Семенов, М. Вилландер, Писъма в ЖЭТФ, 84:3 (2006), 176.

[5] J. Kasprzak, M. Richard, S. Kundermann at al., Nature, 443 (2006), 409.

[6] Ю. Е. Лозовик, О. Л. Берман, ЖЭТФ, 111 (1997), 1879; Yu. E. Lozovik, O. L. Berman, V. G. Tsvetus, Phys. Rev. B, 59 (1999), 5627.

[7] J. M. Kosterlitz, D. J. Thouless, J. Phys. C, 6 (1973), 1181; J. M. Kosterlitz, J. Phys. C, 7 (1974), 1046.

[8] В. Н. Попов, Континуальные интеграль в квантовой теории поля и статистической физике, Атомиздат, М., 1976.

[9] L. Pitaevskii, S. Stringari, Bose-Einstein Condensation, Int. Ser. Monogr. Phys., 116, Clarendon Press, Oxford, 2003.

[10] D. R. Nelson, J. M. Kosterlitz, Phys. Rev. Lett., 39 (1977), 1201.

Поступила в редакцию 30.01.2007 